\title{
Alpha Particle Slowing-Down Characteristics and the Effect on MHD Instability Excitation at High-Density Operation Points in FFHRs ${ }^{*}$
}

\author{
Hideaki MATSUURA, Osamu MITARAI ${ }^{1)}$, Akio SAGARA ${ }^{2)}$ and Yasuyuki NAKAO \\ Department of Applied Quantum Physics and Nuclear Engineering, \\ Kyushu University, 744 Motooka, Fukuoka 819-0395, Japan \\ ${ }^{1)}$ Liberal Arts Education Center, Kumamoto Campus, Tokai University, \\ 9-1-1 Toroku, Kumamoto 862-8652, Japan \\ ${ }^{2)}$ National Institute for Fusion Science, 322-6 Oroshi-cho, Toki, 509-5292, Japan
}

(Received 6 December 2010 / Accepted 27 April 2011)

\begin{abstract}
Alpha-particle slowing-down behaviors at low-temperature, high-density operation points in force-free helical reactors (FFHRs) are examined on the basis of a Fokker-Planck (FP) simulation that simultaneously consider the balance among generation, slowing down, and loss from the plasma in parallel with the density dependence of the Alfvén speed. An accurate treatment of the boundary velocity region between thermal and non-thermal components is shown to be important in evaluating the alpha particle population that can induce instability. In a typical high-density, low-temperature operation point in an FFHR, this population is reduced.
\end{abstract}

(C) 2011 The Japan Society of Plasma Science and Nuclear Fusion Research

Keywords: alpha-particle velocity distribution function, Fokker-Planck equation, Alfvén eigenmode, MHD instability, force-free helical reactor (FFHR)

DOI: $10.1585 /$ pfr.6.2405086

\section{Introduction}

High-density plasma (up to $1.2 \times 10^{21} \mathrm{~m}^{-3}$ ) has been observed in pellet injection experiments at the Large Helical Device (LHD) [1]. High-density, low-temperature operation reduces the divertor heat flux by increased bremsstrahlung radiation $[2,3]$ and ameliorates the pellet penetration problem. Finding a method of controlling the thermally unstable plasma at this operation point has been a major problem. It was recently found that the thermal instability could be stabilized by means of a proportional integral derivative (PID) control algorithm based on fusion power measurement $[2,4]$. To realize high-density, low-temperature operation in a force-free helical reactor (FFHR), the physical characteristics of alpha-particle behavior in such an operation mode should be understood.

In this paper, we focus on alpha particle slowingdown behaviors and the correlation with Alfvén instability excitation in a typical high-density, low-temperature operation mode in an FFHR. The magnetic fluctuation amplitudes of Alfvén eigenmodes are well known to be strongly related to the beta value of the energetic alpha particles. An LHD experiment [5] revealed that energetic ions with speeds between $\sim 0.5$ and $\sim 2.0$ times the Alfvén speed are strongly related to instability excitation. In the high-density operation mode, the Alfvén speed is

author'se-mail: mat@nucl.kyushu-u.ac.jp

*) This article is based on the presentation at the 20th International Toki Conference (ITC20). reduced inversely proportional to the square root of the ion density, and the velocity range associated with instability excitation involves the boundary velocity region between thermal and non-thermal components. Although the slowing-down time is relatively short in high-density, lowtemperature plasmas, the alpha particle concentration itself and the ratio of the thermal (bulk) component to the total distribution function also depend on other plasma parameters. To accurately estimate the alpha particle population that can induce instability (the beta value of energetic alpha-particles), a Fokker-Planck (FP) calculation should be used, which consider the balance among alpha particle generation, slowing down, and loss from the plasma in parallel with the density dependency of the Alfvén speed. FP simulations show that in a typical high-density, lowtemperature operation point in an FFHR, the alpha particle population that can induce instability is reduced.

\section{Analysis Model}

The Boltzmann-Fokker-Planck (BFP) equation for alpha particles is written as

$$
\begin{aligned}
\frac{\partial f_{\alpha}}{\partial t}= & \sum_{j}\left(\frac{\partial f_{\alpha}}{\partial t}\right)_{j}^{\text {Coulomb }}+\sum_{n}\left(\frac{\partial f_{\alpha}}{\partial t}\right)_{n}^{N I} \\
& +\frac{1}{v^{2}} \frac{\partial}{\partial v}\left(\frac{v^{3} f_{\alpha}}{2 \tau_{\mathrm{c}}^{*}(v)}\right)+S_{\alpha}(v)-\frac{f_{\alpha}}{\tau_{\mathrm{p}}^{*}(v)},
\end{aligned}
$$

where $f_{\alpha}(v)$ is the velocity distribution function of alphaparticles. The first term on the right-hand side of Eq. (1) 
represents the Coulomb collision term [6]. The summation is taken over all background species, i.e., $j=$ deuterons, tritons, alpha-particles and electrons. The collision term is hence non linear, and retains collisions between alpha particles. The deuterons, tritons and electrons are assumed to be Maxwellian with temperature $T$. The second term on the right-hand side of Eq. (1) accounts for nuclear plus interference (NI) scattering [7] of alpha particles by background ions $n$ [8]. We consider NI scattering between 1) alpha particles and deuterons, 2) alpha particles and tritons. The NI cross sections are taken from the work of Perkins and Cullen [7].

The third and fifth terms on the right-hand side of Eq. (1) represent diffusion in velocity space due to thermal conduction and particle transport loss from the plasma respectively. To incorporate the unknown loss mechanism of energetic ions into the analysis, following Bittoni's treatment [9], we simulate the velocity dependence of the energy loss time $\tau_{\mathrm{c}}^{*}(v)$ due to thermal conduction and the particle-loss time $\tau_{\mathrm{p}}^{*}(v)$ by using a dimensionless parameter $\gamma$, i.e.,

$$
\tau_{\mathrm{c}(\mathrm{p})}^{*}(v)=\left\{\begin{array}{cl}
C_{\mathrm{c}(\mathrm{P})} \tau_{\mathrm{c}(\mathrm{P})} & \text { when } v<v_{\text {th }} \\
C_{\mathrm{c}(\mathrm{P})} \tau_{\mathrm{c}(\mathrm{P})}\left(v / v_{\mathrm{th}}\right)^{\gamma} & \text { when } v \geq v_{\mathrm{th}}
\end{array} .\right.
$$

The chosen high exponent $\gamma$ ensures rapid increment of both confinement times in higher energy ranges compared with that in the thermal energy range, and thus, hardly any energetic particles and their energy are evacuated compared with thermal particles. For all the calculations in this paper, we assume $\gamma=4$. As discussed in Ref. 8, in typical plasma conditions, $\gamma$ would not be a significant parameter if we choose sufficiently large values, e.g., $\gamma \geq 4$. Considering the energy loss mechanisms resulting from both thermal conduction and particles transport loss from plasma, the global energy confinement time is defined as $1 / \tau_{\mathrm{E}}=1 / \tau_{\mathrm{C}}+1 / \tau_{\mathrm{P}}$. (see Ref. 8 ).

The source $\left[S_{\mathrm{a}}(v)\right]$ and particle loss $\left[L_{\mathrm{a}}(v)\right]$ terms are described so that generation due to $\mathrm{T}(\mathrm{d}, \mathrm{n})^{4} \mathrm{He}$ reactions and transport loss balance each other:

$$
S_{\alpha}(v)-L_{\alpha}(v)=\frac{S_{\alpha}}{4 \pi v^{2}} \delta\left(v-v_{0}\right)-\frac{f_{\alpha}(v)}{\tau_{\mathrm{p}}^{*}(v)} .
$$

Here $v_{0}$ represents the speed corresponding to the alpha particle generation energy, i.e., $3.5 \mathrm{MeV}$. The alpha particle generation rate $S_{\alpha}$ is written as $S_{\alpha}=n_{\mathrm{D}} n_{\mathrm{T}}<\sigma \mathrm{V}>_{\mathrm{DT}}$

\section{Results and Discussion}

Figure 1 shows typical temporal behaviors of the alpha particle distribution functions in several background density plasmas, i.e., (a) $n_{\mathrm{D}}=n_{\mathrm{T}}=1.6 \times 10^{19}$, (b) $8 \times$ $10^{19}$ and (c) $4 \times 10^{20} \mathrm{~m}^{-3}$. We assume that alpha particles production by $\mathrm{T}(\mathrm{d}, \mathrm{n})^{4} \mathrm{He}$ fusion reactions begins at $t$ $=0$. During the simulations, the fuel-ion densities, electron temperature $\left(T \equiv T_{\mathrm{i}}=T_{\mathrm{e}}=10 \mathrm{keV}\right)$, and energy and particle confinement time $\left(\tau_{\mathrm{E}}=(1 / 2) \tau_{\mathrm{p}}=3 \mathrm{~s}\right)$ are kept constant. The slowing-down distribution is formed gradually and at $\sim 10 \mathrm{~s}$ after the beginning of the simulation, it reaches a near equilibrium state. In low-density plasmas, the averaged Coulomb collision frequency between alpha and background charged particles is relatively low compared with that in a high-density plasma. As revealed by the conventional relaxation-time scaling, $\tau_{\text {relax }} \propto T^{3 / 2} / n$, the alpha particle distribution function changes shape more slowly toward the equilibrium state. Note also that in the equilibrium state, the relative magnitudes of the bulk and suprathermal distribution components are strongly influenced by the background density. In high-density plasmas, the ratio of the energetic component to the total distribution function tends to be lower. In this case, the ratios of the component above $3 v_{\text {th }}$ to the total distribution function are $26 \%, 5.3 \%$, and $1.0 \%$ for (a) $n_{\mathrm{D}}=n_{\mathrm{T}}=1.6 \times 10^{19}$, (b) $8 \times 10^{19}$, and (c) $4 \times 10^{20} \mathrm{~m}^{-3}$, respectively.

The typical operation parameter ranges planned for the next-generation helical devices [10], e.g., FFHR2m2,
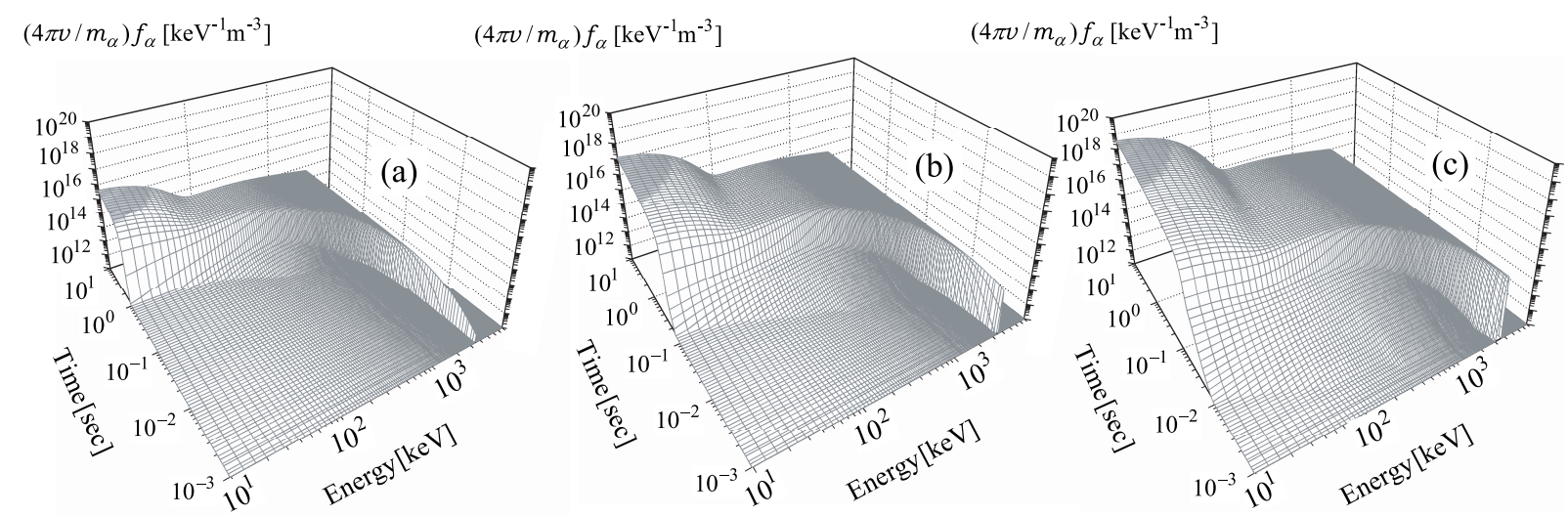

Fig. 1 Time evolution of alpha-particle distribution functions for (a) $n_{\mathrm{D}}=n_{\mathrm{T}}=1.6 \times 10^{19} \mathrm{~m}^{-3}$, (b) $8 \times 10^{19} \mathrm{~m}^{-3}$ and (c) $4 \times 10^{20} \mathrm{~m}^{-3}$ fuel-ion density plasmas. In the calculations, the fuel-ion and electron temperatures are assumed to be $10 \mathrm{keV}$, and the confinement times are assumed to be $\tau_{\mathrm{E}}=(1 / 2) \tau_{\mathrm{p}}=3 \mathrm{~s}$. 
Table 1 Typical operation regions in FFHR2m2 [10], (a) lowdensity, high-temperature region, (b) high-density, lowtemperature region.

\begin{tabular}{ccc}
\hline & (a) low density & (b) high density \\
\hline$T_{\mathrm{e}}[\mathrm{keV}]$ & 18.0 & 6.8 \\
$n_{\mathrm{e}}\left[\mathrm{m}^{-3}\right]$ & $1.8 \times 10^{20}$ & $9.3 \times 10^{20}$ \\
$n_{\mathrm{D}}, n_{\mathrm{T}}\left[\mathrm{m}^{-3}\right]$ & $8.0 \times 10^{19}$ & $4.0 \times 10^{20}$ \\
$\tau_{\mathrm{E}}[\mathrm{s}]$ & 2.3 & 6.0 \\
$B_{\mathrm{T}}[\mathrm{T}]$ & 4.5 & 4.5 \\
$<\beta>[\%]$ & 5.0 & 4.7 \\
$P_{\mathrm{f}}[\mathrm{GW}]$ & 3.0 & 3.0 \\
$\alpha_{\mathrm{T}}$ & 1.0 & 1.0 \\
$\alpha_{\mathrm{n}}$ & 0.5 & 3.0 \\
\hline
\end{tabular}

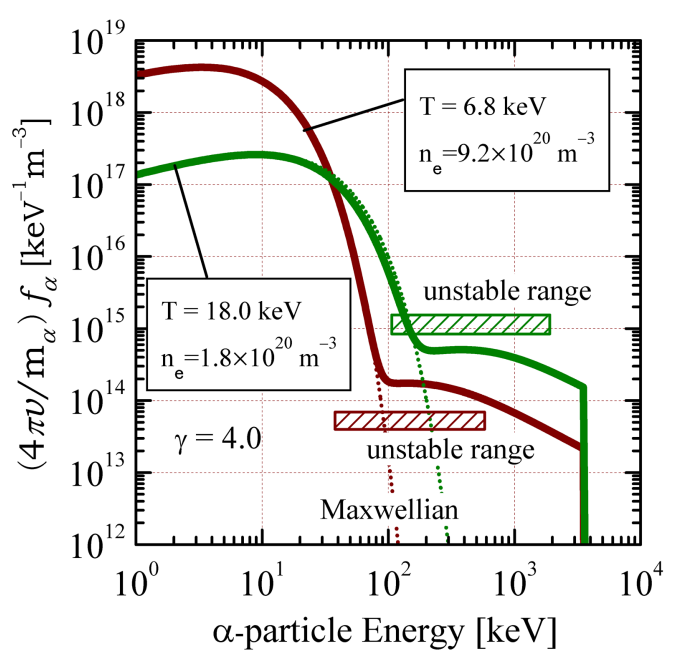

Fig. 2 Alpha-particle distribution functions in typical FFHR operation ranges (see Table 1).

are presented in Table 1. Two types of operation scenario are considered for FFHR2m2: (a) low-density, hightemperature (thermally stable) and (b) high-density, lowtemperature (thermally unstable) operation points. In the table, $\alpha_{\mathrm{n}}\left(\alpha_{\mathrm{T}}\right)$ is a dimensionless parameter introduced to adjust the spatial density (temperature) profile, i.e., $n_{\mathrm{e}}(r)=$ $n_{\mathrm{e}}(0)\left(1-r^{2} / a^{2}\right)^{\alpha_{\mathrm{n}}}\left[T_{\mathrm{e}}(r)=T_{\mathrm{e}}(0) \times\left(1-r^{2} / a^{2}\right)^{\alpha_{\mathrm{T}}}\right]$. Here, $n_{\mathrm{e}}(0)\left[T_{\mathrm{e}}(0)\right]$ is the electron density (temperature) at $r=0$, and $a$ represents the minor radius $(a=2.5 \mathrm{~m})$. In the subsequent discussion, the alpha particle distribution function is examined in these two typical operation ranges.

Figure 2 shows the equilibrium alpha particle distribution functions for these two typical operation scenarios. The dotted lines represent a Maxwellian at the same temperature as the bulk alpha-particles. As noted above, the slowing-down effect is enhanced in higher-density, lowertemperature plasmas. The concentration of alpha particles, however, is a few times larger in the high-density operation region. The slowing-down distribution is determined by the competition between these effects. The magnitude of the energetic component of the alpha particle distribu-

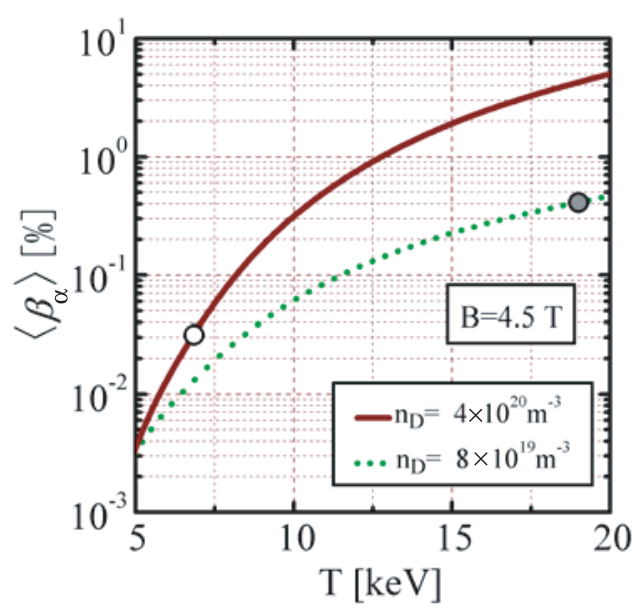

Fig. $3\left\langle\beta_{\alpha}\right\rangle$ parameter as a function of temperature for typical densities at FFHR operation points. White and black circles represent high- and low-density operation points, respectively, in Table 1.

tion function at the high-density operation point is several times smaller than that at the low-density operation point.

It is well known that alpha particles having a velocity nearly equal to the Alfvén velocity can induce several types of plasma instability [5]. Note that the Alfvén speed itself is also inversely proportional to the square root of the ion density. The relative intensity of energetic alpha particles that can induce instability depends on the competition between the acceleration of the slowing-down process and the decrement in the Alfvén speed due to the increased density. Energetic ions with speed between $\sim 0.5$ and $\sim 2.0$ times the Alfvén speed was reported to be strongly related to instability excitation [5]. The energy range from $\sim 100 \mathrm{keV}$ to $\sim 2 \mathrm{MeV}[\sim 30 \mathrm{keV}$ to $\sim 400 \mathrm{keV}]$ corresponds to the above Alfvén speed range in the (a) low-density, high-temperature [(b) high-density, low-temperature] operation point indicated in Table 1. In Fig. 2, the number of alpha particles included in these unstable energy ranges shows a rough tendency to be somewhat smaller in the high-density plasma than in the low-density plasma.

To quantitatively examine the relative intensity of alpha particles that can induce instabilities in the entire plasma volume, the parameter $\left\langle\beta_{\alpha}\right\rangle$, which is the volume averaged beta value for energetic alpha particles, is introduced,

$$
\left\langle\beta_{\alpha}\right\rangle \equiv \frac{\frac{4}{3} \pi \int_{v_{\mathrm{A}} / 2}^{2 v_{\mathrm{A}}} m_{\alpha} v^{4} f_{\alpha} d v}{B_{\mathrm{T}}^{2} / 2 \mu_{0}\left(1+\alpha_{\mathrm{n}}+\alpha_{\mathrm{T}}\right)},
$$

where $v_{\mathrm{A}}$ represents the Alfvén speed, and $B_{\mathrm{T}}$ is the toroidal field. In Eq. (4), the lower (upper) limit for alpha particle speed in the integration is set to half (twice) the Alfvén speed. In Fig. 3, the $\left\langle\beta_{\alpha}\right\rangle$ parameter is shown as a function of temperature. The solid line represents the results when $n_{\mathrm{D}}=4 \times 10^{20} \mathrm{~m}^{-3}$ [which includes the high 


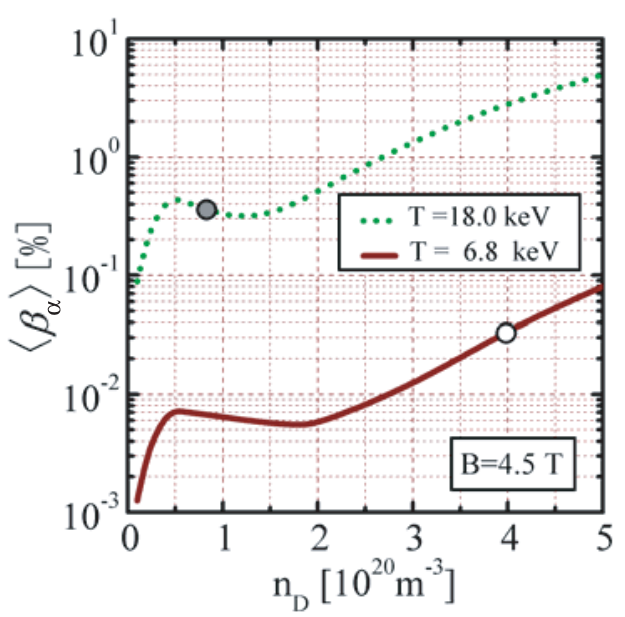

Fig. $4\left\langle\beta_{\alpha}\right\rangle$ parameter as a function of deuteron density for typical temperatures at FFHR operation points. White and black circles represent high- and low-density operation points, respectively, in Table 1.

density operation point in Table 1 (white circle)], and the dotted line denotes the results when $n_{\mathrm{D}}=8 \times 10^{19} \mathrm{~m}^{-3}$ [which includes low-density operation point in Table 1 (black circle)]. For the $\left\langle\beta_{\alpha}\right\rangle$ parameter on isothermal line, the energetic component related to instability excitation in the alpha particle distribution function increases in higherdensity plasmas. However, the difference between the two curves in Fig. 3 is considerably smaller in the lowtemperature region. This is because the acceleration of the slowing-down process is highly enhanced with the decreasing temperature, and in the low-temperature range, the unstable energy range belongs to the suprathermal component of the alpha particle distribution function.

Next, we show the $\left\langle\beta_{\alpha}\right\rangle$ parameter as a function of deuteron density (Fig. 4). The solid line represents the results for $T=6.8 \mathrm{keV}$ [which includes the high-density operation point in Table 1 (white circle)], and the dotted line denotes the results for $T=18.0 \mathrm{keV}$ [which includes the low-density operation point in Table 1 (black circle)]. As described above, the density dependency of the $\left\langle\beta_{\alpha}\right\rangle$ parameter depends on the competition among (a) the increment (decrement) of the alpha particle density, i.e., the alpha article generation rate, (b) the acceleration (reduction) of the slowing-down effect, and (c) the decrement (increment) of the Alfvén speed due to increased (decreased) ion density. Over the entire range, the $\left\langle\beta_{\alpha}\right\rangle$ parameter tends to increase with increasing density owing to the increased fusion reaction rate. At densities of $\sim 5 \times 10^{19}$ to $\sim 2 \times$
$10^{20} \mathrm{~m}^{-3}$, however, the curves are almost steady. This is due to the acceleration of the slowing-down effect. At densities above $\sim 2 \times 10^{20} \mathrm{~m}^{-3}$, the Alfvén speed approaches the marginal region between thermal and suprathermal energies, and $\left\langle\beta_{\alpha}\right\rangle$ gradually increases with the increasing density. The $\left\langle\beta_{\alpha}\right\rangle$ parameter is reduced by almost one order of magnitude owing to the temperature decrement over the entire density range. The estimated $\left\langle\beta_{\alpha}\right\rangle$ parameter is $0.035 \%(0.37 \%)$ at the high-density (low-density) operation point shown in Table 1.

\section{Concluding Remarks}

We showed that in the high-density, low-temperature operation range in FFHR2m2, the alpha particle population that can induce instability (the $\left\langle\beta_{\alpha}\right\rangle$ parameter) is reduced by almost one order of magnitude compared with that at the low-density operation point. In this paper, as a first step, we solved only the BFP equation for alpha-particles. When we evaluated the alpha particle distribution function, we did not consider the effects of the instabilities. If a severe instability is induced at some points in the operation parameter ranges, alpha particles may be lost because of the instability. This might reduce the $\left\langle\beta_{\alpha}\right\rangle$ parameter to some extent. A consistent simulation of this point is our next step. The simulation was made on the basis of the BFP model ascertained by comparing the derived fusion product spectrum with previous data [11]. Throughout the calculations the large angle scattering effect has been considered; however, its effect on the present discussion would be small.

\section{Acknowledgment}

The authors acknowledge helpful comments and discussions with Prof. Y. Todo at the NIFS.

[1] N. Ohyabu et al., Phys. Rev. Let. 97, 055002 (2006).

[2] O. Mitarai et al., Plasma Fusion Res. 2, 021 (2007).

[3] A. Sagara et al., Fusion Eng. Des. 83, 1690 (2008).

[4] O. Mitarai et al., Fusion Sci. Technol. 56, 1495 (2009).

[5] S. Yamamoto et al., Nucl. Fusion 45, 326 (2005).

[6] M.N. Rosenbluth et al., Phys. Rev. 107, 1 (1957).

[7] S.T. Perkins and D.E. Cullen, Nucl. Sci. Eng. 20, 77 (1981).

[8] H. Matsuura and Y. Nakao, Phys. Plasmas 13, 062507 (2006).

[9] E. Bittoni et al., Nucl. Fusion 29, 931 (1980).

[10] O. Mitarai et al., 23th IAEA FEC 2010, FTP/P6-19 (2010).

[11] H. Matsuura and Y. Nakao, Phys. Plasmas 16, 042507 (2009). 